Revista do Departamento de Geografia
Universidade de São Paulo
www.revistas.usp.br/rdg
ISSN 2236-2878
V.34 (2017)

\title{
Influência do Sistema de Corte e Queima na Infiltração e Condutividade Hidráulica do Solo
}

\section{Influence of Slash-and-Burn in Infiltration and Soil Hydraulic Conductivity}

\author{
Adalberto Alves Pereira \\ Universidade Estadual do Centro-Oeste \\ adalbertoalvespereira@yahoo.com.br \\ Edivaldo Lopes Thomaz \\ Universidade Estadual do Centro-Oeste \\ ethomaz@brturbo.com
}

Recebido (Received): 26/03//2016 Aceito (Accepted): 05/08/2017

DOI: $10.11606 /$ rdg.v34i0.128859

Resumo: Coivara é um dos sistemas agrícolas mais antigos utilizados pelo homem. Os efeitos deste sistema sobre as propriedades físico-hídricas do solo ainda são controversos. Desta forma, este estudo tem por objetivo avaliar a infiltração e a condutividade hidráulica em coivara sob um ciclo de 2 anos de pousio e comparar os parâmetros hidráulicos da agricultura de coivara com outros sistemas de manejo. Este trabalho foi realizado entre os meses de dezembro de 2012 e janeiro de 2013, em três áreas cultivadas com feijão e uma área de floresta. Realizaram-se 18 ensaios de infiltração utilizando-se um infiltrômetro de tensão a disco em cada área, sendo nove sem aplicação de tensão e nove com tensão de cinco centímetros de água. A coivara apresentou taxas de infiltração e condutividade hidráulica elevadas, semelhantes ao plantio mínimo. Conclui-se, que a manutenção da estrutura do solo é essencial para o movimento da água no solo, e foi fundamental para que a coivara e o plantio mínimo apresentassem taxas de infiltração e condutividade hidráulica superiores ao plantio convencional.

Palavras-Chave: Movimento da Água No Solo; Sistemas de Manejo; Física do Solo; Sustentabilidade.

\begin{abstract}
Slash-and-burn is one of the oldest agricultural systems used by man. The effects of this system on the physical and hydraulic properties of the soil are still controversial. Thus, this study aims to evaluate the infiltration and hydraulic conductivity in slash-and-burn in a cycle of two years of fallow and compare the hydraulic parameters of swidden agriculture with others tillage system. This work was carried out between the months of December 2012 and January 2013 in three areas tillage with beans and a forest area. They were carried out 18 infiltration tests using a drive voltage infiltrometer in each area, nine without application of voltage and nine with a voltage five centimeters of water. The slash and burn presented high rates of infiltration and hydraulic conductivity, similar to the minimum tillage. It is concluded that soil structure is essential for the soil water movement, and it was fundamental for the slash and burn and the minimum tillage to showed rates of infiltration and hydraulic conductivity superior to conventional tillage.
\end{abstract}

Keywords: Soil Water Movement; Tillage System; Soil Physics; Sustainability. 


\section{INTRODUÇÃO}

Coivara é um dos sistemas agrícolas mais antigos utilizados pelo homem (CERRI, 2007). Apesar do surgimento de sistemas agrícolas modernos (por exemplo, plantio direto), ainda persiste em muitas partes do mundo, particularmente nas regiões tropicais (THOMAZ, 2009; ZIEGLER et al., 2009; GROGAN et al, 2012.; VAN VLIET et al, 2012).

Por ser praticado em sua maioria por pequenos agricultores, o sistema de coivara ocupa solos menos produtivos, localizados em encostas íngremes ou fundos de vale. Este sistema (coivara) segue ciclos entre cultivo e pousio, que duram entre 3 e 5 anos, as fases de um ciclo completo, são: limpeza, queima, cultivo, abandono e recuperação (THOMAZ, 2009).

Os efeitos deste sistema sobre as propriedades físico-hídricas do solo ainda são controversos (LAWRENCE et al, 2010), mas nos últimos anos muitos pesquisadores tem considerado este um sistema não sustentável (STYGER et al., 2007; ARE et al., 2009; THOMAZ et al., 2014), pois seus efeitos benéficos, como: redução de plantas daninhas, liberação imediata de nutrientes (Niemeyer et al. 2005), aumento da atividade biológica e aumento da matéria orgânica parecem ser de curta duração devido aos seus efeitos degenerativos nas propriedades físicas do solo (WUEST et al., 2005; HUBBERT et al., 2006).

Devido ao aumento na pressão de uso da terra o tempo de regeneração (pousio) destas áreas vem sendo reduzido. A redução do período de pousio torna insuficiente o tempo para que a vegetação e o solo se regenerem, o que contribui para o aumento da degradação e erosão do solo, diminuição da estabilidade de agregados e esgotamento de nutrientes do solo (ZIEGLER et al., 2009) reduzindo a produtividade, tornando necessária a incorporação de mais áreas para manter os níveis de colheita necessários.

Mukul and Herbohn (2016) realizaram avaliação de estudos de caso relacionados ao sistema de coivara e verificaram que são poucos os estudos relacionados à hidrologia e física do solo nestas áreas. Desta forma, este estudo tem por objetivo (1) avaliar a infiltração e a condutividade hidráulica em coivara sob um ciclo de 2 anos de pousio, (2) comparar os parâmetros hidráulicos da agricultura de coivara com outros sistemas de manejo.

\section{MATERIAL E MÉTODOS}

Este estudo foi realizado em três áreas cultivadas com feijão preto (Phaseolus vulgaris L.); e uma com floresta (Tabela 1) na bacia hidrográfica do Arroio Palmeirinha, município de Reserva - PR. A escolha da cultura do feijão se deu por ser esta a principal cultura praticada no município, com produção de aproximadamente 28.200 toneladas, tornando-o o terceiro maior produtor do estado do Paraná e o décimo maior do país (SALVADOR, 2011). A área de floresta foi utilizada como área controle.

As áreas estão em altitude variando entre 780 e 820 metros com clima Subtropical úmido (MINEROPAR, 2007) com verões frescos, geadas severas no inverno, sem estação seca, sendo que as temperaturas dos meses mais quentes são inferiores a $22^{\circ} \mathrm{C}$ e dos meses mais frios inferiores a $18^{\circ} \mathrm{C}$ e precipitação entre 1400 e $1800 \mathrm{~mm}$ por ano (ITCG, 2008).

Realizou-se a caracterização físico-química do solo até a profundidade $0,15 \mathrm{~m}$ nas áreas estudadas (Tabela 2). Escolheu-se esta profundidade por ser a mais afetada pelo plantio do feijão (CTSBF, 2012), assim como, a mais afetada pela ação do fogo durante a limpeza da área de coivara (THOMAZ et al., 2014).

Os solos das áreas estudadas foram caracterizados como Argissolos vermelho-amarelo eutrófico (EMBRAPA, 2013), de textura médio argilosa e horizonte A com cerca de 0,2 $\mathrm{m}$ de profundidade. De maneira geral os solos das áreas agrícolas possuem características semelhantes na camada superficial (0,0-0,15 m), especialmente, textura, matéria orgânica e capacidade de troca de cátions. 
Tabela 1. Características de manejo nas áreas estudadas

\begin{tabular}{|c|c|c|}
\hline Área & $\begin{array}{c}\text { Tamanho } \\
\text { (ha) }\end{array}$ & Características de manejo \\
\hline $\begin{array}{l}\text { Plantio } \\
\text { convencional } \\
\text { (PC) }\end{array}$ & 2 & $\begin{array}{l}\text { Vertente retilínea. } 10 \text { anos de cultivo (2002-2012) com rotação entre feijão e } \\
\text { milho, manejo tradicional, uma aração e duas gradagens com implementos } \\
\text { à tração animal para plantio em linha e colheita manual. Declividade variando } \\
\text { entre } 6^{\circ} \text { e } 12^{\circ} \text {. }\end{array}$ \\
\hline $\begin{array}{l}\text { Coivara } \\
\text { (CV) }\end{array}$ & 1 & $\begin{array}{l}\text { Vertente côncava. Primeiro cultivo após } 2 \text { anos de regeneração (2010-2012). } \\
\text { Histórico anterior - rotação milho-feijão. Limpeza da área com utilização de } \\
\text { queima. Declividade entre } 20^{\circ} \text { e } 30^{\circ} \text {. Manejo tradicional com capina, plantio } \\
\text { com matraca e colheita manual. }\end{array}$ \\
\hline $\begin{array}{l}\text { Plantio mínimo } \\
\text { (PM) }\end{array}$ & 5 & $\begin{array}{l}\text { Vertente côncavo-convexa. Plantio direto mínimo, } 30 \text { anos de cultivo com } \\
\text { rotação milho e feijão. Manejo mecanizado para plantio em linha, e colheita } \\
\text { manual. Declividade entre } 6^{\circ} \text { e } 12^{\circ} \text {. }\end{array}$ \\
\hline $\begin{array}{l}\text { Floresta } \\
(\mathrm{FT})\end{array}$ & 2 & $\begin{array}{l}\text { Vertente côncavo-convexa. Floresta secundária, caracterizada pelos } \\
\text { moradores locais como capoeirão, sem dados de alteração no período } 1962- \\
\text { 2012. Declividade entre } 6^{\circ} \text { e } 12^{\circ} \text {. }\end{array}$ \\
\hline
\end{tabular}

Fonte: Autores.

Tabela 2: Propriedades físico-químicas do solo até a profundidade de $0,15 \mathrm{~m}$ nas diferentes áreas avaliadas

\begin{tabular}{cccccccccc}
\hline & $\begin{array}{c}\text { Areia } \\
\left(\mathrm{g} \mathrm{kg}^{-1}\right)\end{array}$ & $\begin{array}{c}\text { Silte } \\
\left(\mathrm{g} \mathrm{kg}^{-1}\right)\end{array}$ & $\begin{array}{c}\text { Argila } \\
\left(\mathrm{g} \mathrm{kg}^{-1}\right)\end{array}$ & $\begin{array}{c}\mathrm{DS} \\
\left(\mathrm{g} \mathrm{cm}^{-3}\right)\end{array}$ & $\begin{array}{c}\text { PT } \\
(\%)\end{array}$ & $\begin{array}{c}\text { DMP } \\
(\mathrm{mm})\end{array}$ & $\begin{array}{c}\mathrm{pH} \\
\left(\mathrm{CaCl}_{2}\right)\end{array}$ & $\begin{array}{c}\text { MO } \\
(\%)\end{array}$ & $\begin{array}{c}\mathrm{CTC} \\
\left(\mathrm{cmol} \mathrm{dm}^{-3}\right)\end{array}$ \\
\hline PC & $442 \pm 6,1^{1}$ & $220 \pm 6,2$ & $338 \pm 4,4$ & $1,14 \pm 0,11$ & $51,1 \pm 5,27$ & $1,54 \pm 0,45$ & $4,62 \pm 0,08$ & $3,07 \pm 0,15$ & $12,04 \pm 0,91$ \\
CV & $438 \pm 4,4$ & $221 \pm 3,6$ & $341 \pm 3,5$ & $1,04 \pm 0,18$ & $53,46 \pm 7,55$ & $2,04 \pm 0,29$ & $5,32 \pm 0,59$ & $3,51 \pm 0,68$ & $12,52 \pm 2,38$ \\
PM & $437 \pm 4,6$ & $221 \pm 2,6$ & $342 \pm 5,0$ & $1,15 \pm 0,12$ & $50,92 \pm 4,59$ & $1,6 \pm 0,72$ & $5,02 \pm 0,29$ & $3,49 \pm 0,18$ & $12,67 \pm 1,53$ \\
FT & $443 \pm 3,5$ & $224 \pm 2,6$ & $333 \pm 5,2$ & $1,00 \pm 0,14$ & $56,05 \pm 6,68$ & $1,96 \pm 0,71$ & $4,84 \pm 0,08$ & $3,76 \pm 0,70$ & $14,76 \pm 2,06$
\end{tabular}

n:24, ${ }^{1}$ média \pm desvio padrão. PC - Plantio convencional; CV - Coivara; PM - Plantio mínimo; FT - Floresta; DS

- Densidade do solo; PT - Porosidade total; DMP - diâmetro médio ponderado; MO - Matéria orgânica; CTC

- Capacidade de troca de cátions. Org. Autores.

Os ensaios de infiltração foram realizados entre os meses de dezembro de 2012 e janeiro de 2013 , utilizando-se um infiltrômetro de tensão a disco com diâmetro de $20 \mathrm{~cm}$. Foram realizados 18 ensaios em cada área, sendo nove sem aplicação de tensão $\left(h_{0}\right)$ e nove com tensão de cinco centímetros de água (h-5).

Para se determinar a taxa de infiltração básica, utilizou-se a Eq. 1 descrita por Pott e De Maria (2003).

$$
V I B=q 60\left(\frac{D t^{2}}{D b^{2}}\right)
$$

Onde,

VIB - Velocidade de infiltração básica $\left(\mathrm{cm} \mathrm{h}^{-1}\right), \mathrm{q}$ - fluxo constante de água do infiltrômetro de tensão $\left(\mathrm{cm} \mathrm{min}{ }^{-1}\right)$, Dt - diâmetro do tubo do infiltrômetro de tensão $(\mathrm{cm}), \mathrm{Db}$ - diâmetro da base do infiltrômetro de tensão $(\mathrm{cm})$.

O fluxo de água no solo está relacionado à condutividade hidráulica, e foi estimado através da Eq. 2, baseada em Wooding (1968).

$$
Q=\pi r^{2} K\left(1+\frac{4}{\pi r \alpha}\right)
$$

Onde,

$\mathrm{Q}$ - fluxo em tridimensional $\left(\mathrm{cm}^{3} \mathrm{~h}^{-1}\right), \mathrm{r}$ - raio do disco $(\mathrm{cm}), \mathrm{K}$ - condutividade hidráulica $\left(\mathrm{cm} \mathrm{h}^{-1}\right), \alpha$ - é uma constante dependente da vazão e da tensão aplicada pelo infiltrômetro $\left(\mathrm{cm}^{-1}\right)$. 
A condutividade hidráulica não saturada foi estimada aplicando-se a Eq. 3, descrita por Gardner (1958).

$$
K(h)=K_{\text {sat }} \exp (\alpha h)
$$

Onde,

$\mathrm{K}(\mathrm{h})$ - condutividade hidráulica não saturada $\left(\mathrm{cm} \mathrm{h}^{-1}\right)$, Ksat - condutividade hidráulica saturada (cm $\mathrm{h}^{-}$ $\left.{ }^{1}\right), \mathrm{h}$ - tensão aplicada pelo infiltrômetro $(\mathrm{cm}), \alpha$ - constante dependente da vazão e da tensão aplicada pelo infiltrômetro $\left(\mathrm{cm}^{-1}\right)$.

Para obtenção da condutividade hidráulica saturada utilizou-se a Eq. 4, descrita também por Gardner (1958).

$$
Q\left(h_{n}\right)=\pi r^{2} K_{s a t} \exp \left(\alpha h_{n}\right)\left(1+\frac{4}{\pi r \alpha}\right)
$$

Onde,

$\mathrm{Q}\left(\mathrm{h}_{\mathrm{n}}\right)$ - volume do fluxo de água do infiltrômetro sob determinada tensão $\left(\mathrm{cm}^{3} \mathrm{~h}^{-1}\right), \mathrm{r}$ - raio do disco $(\mathrm{cm}), \mathrm{h}_{\mathrm{n}}$ - tensão exercida pelo infiltrômetro $(\mathrm{cm}), \mathrm{K}_{\text {sat }}$ - condutividade hidráulica saturada $\left(\mathrm{cm} \mathrm{h}^{-1}\right)$.

De acordo com a tensão aplicada pelo infiltrômetro utilizando a equação da capilaridade (Eq. 5) pode se determinar o tamanho dos poros que participam do processo de infiltração.

$$
r=\frac{-2 \sigma \cos \alpha}{\rho g h}
$$

Onde,

$r$ - raio do poro $(\mathrm{mm}), \sigma$ - tensão da água $\left(\mathrm{N} \mathrm{m}^{-1}\right)$, cos $\alpha$ - Cosseno do ângulo de contato entre a água e a parede do poro (utiliza-se o valor de 1, que representa contato perfeito), $\rho$ - densidade da água $\left(\mathrm{kg} \mathrm{m}^{-3}\right), \mathrm{g}$ - gravidade $\left(\mathrm{m} \mathrm{s}^{-2}\right), \mathrm{h}$ - tensão de água no infiltrômetro $(\mathrm{mm})$.

Utilizando a equação de Poiseuille (Eq. 6) obtêm-se a estimativa do número de poros de raio r por $\mathrm{m}^{2}$ de solo (HILLEL, 1998).

$$
N=\frac{7,2210^{-2} \Delta K}{r^{4}}
$$

Onde,

$\mathrm{N}$ - número de poros de raio $\mathrm{r}$ por $\mathrm{m}^{2}$ de solo, $\Delta \mathrm{K}$ - variação de condutividade hidráulica de acordo com a tensão aplicada pelo infiltrômetro $(\mathrm{mm}), \mathrm{r}$ - raio do poro $(\mathrm{mm})$.

Para análise estatística, em princípio realizou-se análise descritiva (média, desvio padrão, coeficiente de variação) e avaliação da distribuição dos dados. Os dados foram submetidos à análise de variância (ANOVA), utilizando-se delineamento inteiramente casualizado. A comparação de médias foi feita pelo teste de Tukey, ao nível de $5 \%$.

Aplicou-se análise de agrupamento hierárquico (cluster - estatística multivariada) buscando dividir o conjunto de áreas em grupos homogêneos (McGARIGAL et al., 2000). Os resultados desta análise foram plotados em um dendograma, demonstrando a distância euclidiana entre as áreas com base nas propriedades físico-hídricas (infiltração acumulada, infiltração inicial, infiltração final, condutividade hidráulica saturada e não saturada, proporção $\mathrm{K}_{-5} / \mathrm{K}_{0}$ e número de poros $>0,3 \mathrm{~mm}$ por $\left.\mathrm{m}^{2}\right)$. 


\section{RESULTADOS E DISCUSSÃO}

A infiltração inicial não diferiu entre as formas de manejo e tensões aplicadas (Tabela 3). Contudo, sem aplicação de tensão a infiltração final e acumulada foram maiores no sistema de coivara. Igualmente, com aplicação de tensão as taxas de infiltração não diferiram entre as formas de manejo. A floresta por apresentar alta umidade antecedente ( $35 \%$; outras áreas $\sim 22 \%)$ teve infiltração reduzida em ambas às tensões aplicadas.

Tabela 3: Taxas de infiltração sob diferentes tensões aplicadas.

\begin{tabular}{lcccccc}
\hline \multirow{2}{*}{ Área } & \multicolumn{3}{c}{ ho } & \multicolumn{3}{c}{$\mathrm{h}-5$} \\
\cline { 2 - 7 } & $\mathrm{li}\left(\mathrm{cm} \mathrm{h}^{-1}\right)$ & $\mathrm{Fi}\left(\mathrm{cm} \mathrm{h}^{-1}\right)$ & $\mathrm{Ci}(\mathrm{cm})$ & $\mathrm{li}\left(\mathrm{cm} \mathrm{h}^{-1}\right)$ & $\mathrm{Fi}\left(\mathrm{cm} \mathrm{h}^{-1}\right)$ & $\mathrm{Ci}(\mathrm{cm})$ \\
\hline $\mathrm{PC}$ & $3,38 \pm 1,11^{1} \mathrm{~A}^{2}$ & $0,31 \pm 0,03 \mathrm{~B}$ & $16,59 \pm 2,21 \mathrm{~B}$ & $1,21 \pm 0,31 \mathrm{~A}$ & $0,20 \pm 0,09 \mathrm{~A}$ & $10,48 \pm 3,09 \mathrm{~A}$ \\
$\mathrm{CV}$ & $4,19 \pm 1,29 \mathrm{~A}$ & $0,67 \pm 0,16 \mathrm{~A}$ & $30,8 \pm 6,64 \mathrm{~A}$ & $1,06 \pm 0,48 \mathrm{AB}$ & $0,19 \pm 0,11 \mathrm{AB}$ & $8,42 \pm 4,12 \mathrm{AB}$ \\
$\mathrm{PM}$ & $4,67 \pm 0,75 \mathrm{~A}$ & $0,63 \pm 0,33 \mathrm{AB}$ & $27,4 \pm 9,02 \mathrm{AB}$ & $1,87 \pm 0,41 \mathrm{~A}$ & $0,19 \pm 0,03 \mathrm{~A}$ & $11,34 \pm 1,46 \mathrm{~A}$ \\
$\mathrm{FT}$ & $4,20 \pm 1,98 \mathrm{~A}$ & $0,31 \pm 0,17 \mathrm{~B}$ & $15,72 \pm 8,01 \mathrm{~B}$ & $0,59 \pm 0,01 \mathrm{~B}$ & $0,05 \pm 0,01 \mathrm{~B}$ & $4,17 \pm 1,84 \mathrm{~B}$ \\
\hline
\end{tabular}

${ }^{1}$ média \pm desvio padrão. ${ }^{2}$ Letras iguais na mesma coluna não diferem ao nível de $5 \%$ no teste de Tukey. $h_{0}-$ taxa de infiltração sem aplicação de tensão; $h_{-5}$ - taxa de infiltração com tensão de $5 \mathrm{~cm}$ de água; li - Infiltração inicial; Fi - Infiltração final; Ci - Infiltração acumulada; PC - Sistema convencional; CV - Coivara; PM - Plantio mínimo; FT - Floresta. Org. Autores.

A coivara e o plantio mínimo mantêm preservada a estrutura do solo, devido ao baixo grau de revolvimento e elevado acúmulo de matéria orgânica no topo do solo. Portanto, contribuem para continuidade e conectividade dos poros, contribuindo para infiltração elevada.

A condutividade hidráulica saturada $\left(\mathrm{K}_{0}\right)$ foi elevada em coivara e plantio mínimo (Figura 1). Silva et al. (2012) encontraram valores médios de $7,84 \mathrm{~cm} \mathrm{~h}^{-1}$ de condutividade hidráulica em Argissolos sob sistemas conservacionistas de manejo, valores estes, muito próximos dos encontrados nas áreas CV e PM.

Plantio convencional apresentou a maior proporção $\mathrm{K}_{-5} / \mathrm{K}_{0}(59.52 \%)$, reduzindo na sequência Floresta>Coivara>Plantio mínimo (Figura 2). Com aplicação de tensão reduz-se o tamanho dos poros que participam do processo de infiltração (SAUER et al., 1990). Desta forma, quanto maior a proporção $\mathrm{K}_{-5} / \mathrm{K}_{0}$, menor a quantidade e a influência dos poros com tamanho superior a $0,3 \mathrm{~mm}$ no fluxo da água.

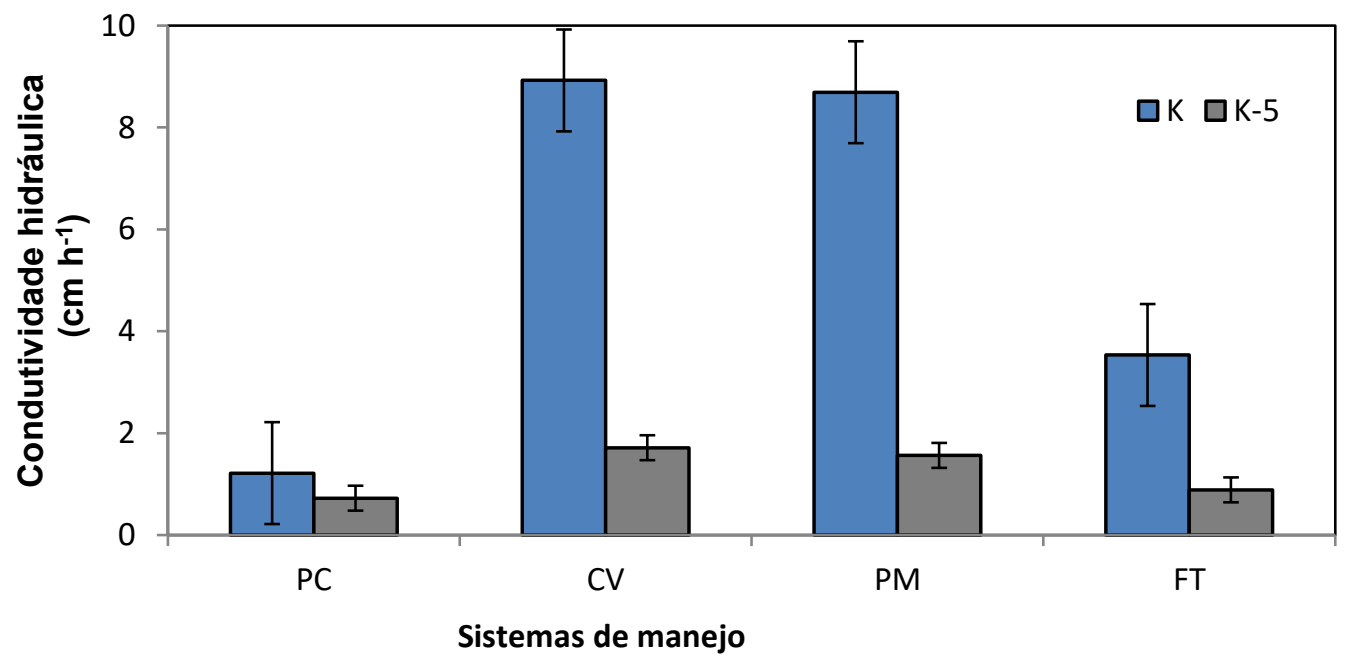

Figura 1. Condutividade hidráulica em diferentes sistemas de manejo e tensões aplicadas. $\mathrm{K}-$ Condutividade hidráulica saturada; K-5 - Condutividade hidráulica em tensão de $-5 \mathrm{~cm}$ de água; PC - Plantio convencional; CV - Coivara; PM - Plantio mínimo; FT - Floresta. Org. Autores. 


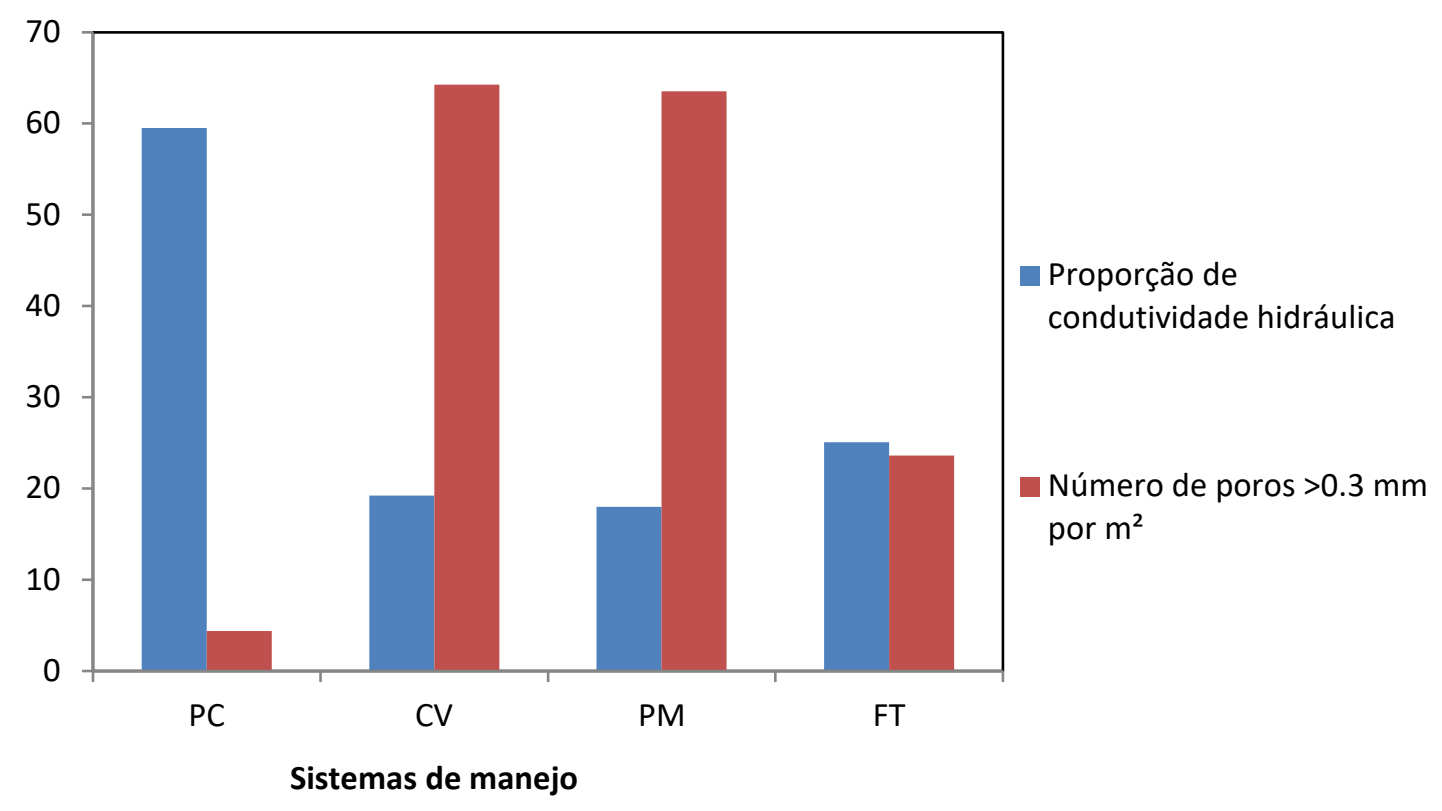

Figura 2. Proporção $\mathrm{K}_{-5} / \mathrm{K}$ e número de poros em diferentes sitemas de manejo. PC - Plantio convencional; CV - Coivara; PM - Plantio mínimo; FT - Floresta. Org. Autores.

Por serem variáveis intimamente relacionadas (condutividade hidráulica e macroporosidade), com a redução da macroporosidade ocorre redução da condutividade hidráulica saturada, uma vez que os macroporos são os principais condutores de água no solo em condições saturadas (HILLEL 1998; BEVEN e GERMANN 2013; PREVEDELLO et al. 2013). Enquanto os microporos funcionam na redistribuição e retenção da água no solo.

O sistema convencional rompe a camada superficial através do uso de equipamentos para aração e gradagem do solo, reduzindo os agregados (Tabela 2) em partículas finas, com o rearranjamento destas partículas que vão preenchendo os poros do solo, tem-se a redução da macroporosidade.

Agregados maiores contribuem para maior macroporosidade do solo. Observamos que este sistema (coivara) é rico em grandes agregados ( $\geq 2 \mathrm{~mm}$ ) (Tabela 2). Além disto, a vegetação de capoeira apresenta rede de raízes bem desenvolvidas que após corte e queima se decompõem, deixando canais preferenciais para o fluxo de água.

$\mathrm{Na}$ agricultura de coivara o fogo utilizado para limpeza da área pode afetar a agregação das partículas devido à recristalização dos óxidos de ferro e alumínio (CERTINI, 2005; SHAKESBY e DOERR, 2006; FOX et al., 2007), no entanto no presente estudo não verificamos perda na estrutura do solo que comprometesse o processo de infiltração (ARE et al., 2009; THOMAZ et al., 2014).

A Figura 3 sintetiza a relação entre as formas de manejo e as propriedades hidráulicas do solo. Verifica-se que os sistemas de coivara e plantio mínimo apresentam elevada semelhança das propriedades físico hidráulicas, próximo dos parâmetros obtidos na área de floresta. $\mathrm{O}$ sistema convencional por alterar significativamente a estrutura do solo apresenta os parâmetros hidráulicos mais desfavoráveis.

As práticas com baixo grau de revolvimento do solo, independentes do tempo de manejo, vêm contribuindo para a melhoria dos parâmetros hidráulicos, tornando estes superiores aos valores da área de floresta.

Estes dados corroboram com Silva et al., (2005), que verificaram melhores condições físicohídricas do solo em sistemas conservacionistas, os quais têm como premissa o não revolvimento ou a mínima mobilização do solo, bem como a manutenção dos resíduos culturais na superfície ou sua incorporação apenas parcial. 


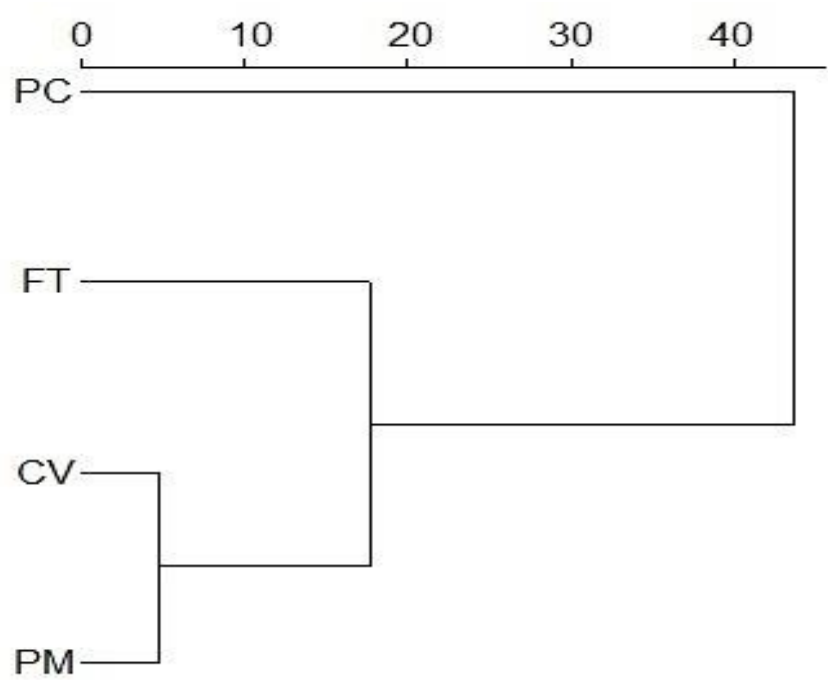

Figura 3. Análise de grupamento (Cluster) com base nos parâmetros hidráulicos do solo. PC - Plantio convencional; CV - Coivara; PM - Plantio mínimo; FT - Floresta. Org. Autores.

\section{CONCLUSÕES}

A coivara apresentou taxas de infiltração e condutividade hidráulica elevadas, semelhantes ao plantio mínimo. Conclui-se também, que a manutenção da estrutura do solo é essencial para o movimento da água no solo, e foi fundamental para que a coivara e o plantio mínimo apresentassem taxas de infiltração e condutividade hidráulica superiores ao plantio convencional.

O sistema convencional por romper a camada superficial do solo altera sua estrutura, refletindo de maneira significativa na sua hidrologia, com redução das taxas de infiltração e condutividade hidráulica.

\section{REFERÊNCIAS}

ARE, K. S.; OLUWATOSIN, G. A.; ADEYOLANU, O. D.; OKE, A. O.. Slash and burn effect on soil quality of na Alfisol: Soil physical properties. Soil Tillage Research. v. 103, p.4-10, 2009.

BEVEN, K.; GERMANN, P.. Macropores and water flow in soils revisited. Water Resources Research. v.49, p.3071-3092, 2013.

CERRI, C. E. P.; SPAROVEK, G.; BERNOUX, M.; EASTERLING, W. E.; MELILLO, J. M.; CERRI, C. C.. Tropical agriculture and global warming: impacts and mitigation options. Scientia Agrícola, v.64, p.83-99, 2007.

CERTINI, G. Effects of fire on properties of forest soils: a review. Oecologia, n.143, p.1-10, 2005.

COMISSÃO TÉCNICA SUL-BRASILEIRA DE FEIJÃO (CTSBF). Informações técnicas para o cultivo de feijão na Região Sul brasileira. 2.ed. Florianópolis: Epagri, 2012. 157p.

EMBRAPA, Centro Nacional de Pesquisa do Solo. Sistema brasileiro de classificação de solos. 3.ed. Rio de Janeiro: Embrapa Solos, 2013, 353p.

FOX, D.M; DARBOUX F.; CARREGA, P.. Effects of fire-induced water repellency on soil aggregate stability, splash erosion, and saturated hydraulic conductivity for different size fractions. Hydrological Processes. n.21, p.2377-2384, 2007.

GARDNER, W. R.. Some steady state solutions of unsatured moisture flow equations with application to evaporation from a water table. Soil Science, 85, p.228-232, 1958.

GROGAN, P.; LALNUNMAWIA, F.; TRIPATHI, S.K.. Shifting cultivation in steeply sloped regions: a review of management options and research priorities for Mizoram state, Northeast India. Agroforest System. 84, 163-177, 2012.

HILLEL, D. Environmental soil physics. San Diego: Academic Press, 1998. 771 p. 
HUBBERT, K.R.; PREISLER, H.K.; WOHLGEMUTH, P.M.; GRAHAM, R.G.; NAROG, M.G.; Prescribed burning effects on soil physical properties and water repellency in a steep chaparral watershed, Southern California, USA. Geoderma. v.130, 284-298, 2006.

ITCG, Instituto De Terras Cartografia e Geociências. Carta de Climas do Estado do Paraná, Escala 1:250000, 2008. Disponível em www,itcg,pr,gov,br. Acesso em 20 de março de 2012.

KLEINMAN, P. J. A.; PIMENTEL, D.; BRYANT, R. B.. The ecological sustainability of slash-and-burn agriculture. Agriculture, Ecosystem and Environment. n.52, p.235-249, 1997.

LAWRENCE, D.; RADEL, C.; TULLY, K.; SCHMOOK, B.; SCHNEIDE, L.. Untangling a decline in tropical forest resilience: constraints on the sustainability of shifting cultivation across the globe. Biotropica 42, 21-30, 2010.

MCGARIGAL, K. ; CUSHMAN, S. A.; STAFFORD, S. G.. Multivariate statistics for Wildlife and Ecology Research. New York: Springer-Verlag, 2000, 283p.

MINEROPAR, Minerais do Paraná. Mapa de Vulnerabilidade Geoambiental do Estado do Paraná, Escala 1:650000, Curitiba, 2007, Disponível em <www,mineropar,pr,gov,br>, Acesso em: 04 outubro 2012.

MUKUL, S. A.; HERBOHN, J.. The impacts of shifting cultivation on secondary forests dynamics in tropics: A synthesis of the key findings and spatio temporal distribution of research. Environmental Science e Policy, v.55, n.1, p.167-177, 2016.

NIEMEYER, T.; NIEMEYER, M.; MOHAMED, A.; FOTTNER, S.; HARDTLE, W.. Impact of prescribed burning on the nutrient balance of heathlands with particular reference to nitrogen and phosphorus. Applied Vegetation Science. 8, 183-192, 2005.

POTT, C. A.; DE MARIA, I. C.. Comparação de métodos de campo para determinação da velocidade de infiltração básica. Revista Brasileira de Ciência do Solo, v.27, n.1, p. 19-27, 2003.

PREVEDELLO, J.; VOGELMANN, E. S.; KAISER, D. R.; REINERT, D. J.. A funcionalidade do sistema poroso do solo em floresta de eucalipto sob Argissolo. Scientia Forestalis, v.41, n.100, p.557-566, 2013.

SALVADOR, C. A.. Análise da conjuntura agropecuária safra 2011/2012. Secretaria de Agricultura e Abastecimento do Paraná (SEAB-PR). Relatório técnico, 2011. Disponível em www.seab.pr.gov.br. Acesso em março de 2012.

SAUER, T. J.; CLOTHIER, B. E.; DANIEL, T. C.. Surface measurements of the hydraulic properties of a tilled and untilled soil. Soil Tillage Research. v.15, p.359-369, 1990.

SHAKESBY, R.A.; DOERR, S. H.. Wildfire as a hydrological and geomorphological agent. EarthScience Reviews. n.74, p.269-307, 2006.

SILVA, S.; MIELNICZUK, J.; ANANIAS, M.; ALBUQUERQUE, J. A.; MAFRA, A. L.; BAYER, C., Atributos físicos do solo relacionados ao armazenamento de água em um Argissolo Vermelho sob diferentes sistemas de preparo. Ciência Rural, n.35, v.3, p.544-552, 2005.

SILVA, J. R. L. da; MONTENEGRO, A. A. A.; SANTOS, T. E. M. dos. Caracterização física e hidráulica de solos em bacias experimentais do semiárido brasileiro, sob manejo conservacionista. Revista Brasileira de Engenharia Agrícola e Ambiental, v, 16, n, 1, p,27-36, 2012.

STYGER, E.; RAKOTONDRAMASY, H. M.; PFEFFER, M. J.; FERNANDES, E. C. M.; BATES, D. M. Influence of slash-and-burn farming practices on fallow sucession and land degradation in the rainforest region of Madagascar. Agriculture Ecosystems Environment, v.119, p.257-269, 2007.

THOMAZ, E.L. The influence of traditional steep land agricultural practices on runoff and soil loss. Agriculture Ecosystems Environment. 130, p.23-30, 2009.

THOMAZ, E. L.; ANTONELI, V.; DOERR, S. H. Effects of fire on the physicochemical properties of soil in a slash-and-burn agriculture. Catena, 122, p.209-215, 2014.

VAN VLIET, N., MERTZ, O., HEINIMANN, A., LANGANKE, T., PASCUAL, U., SCHMOOK, B., ADAMS, C., SCHMIDT-VOGT, D., MESSERLI, P., LEISZ, S., CASTELLA, J.-C., JØRGENSEN, L., BIRCH-THOMSEN, T., HETT, C., BECH-BRUUN, T., ICKOWITZ, A., VU, K.C., YASUYUKI, K., FOX, J., PADOCH, C., DRESSLER, W., ZIEGLER, A.D. Trends, drivers and impacts of changes in 
swidden cultivation in tropical forest-agriculture frontiers: a global assessment. Global Environmental Change, v.22, p.418-429, 2012.

WOODING, R. A.. Steady infiltration from a shallow circular pond. Water Resource. 4. p.1259-1273, 1968.

WUEST, S.B.; CAESAR-TONTHAT, T.C.; WRIGHT, S. F.; WILLIAMS, J.D. Organic matter addition, $\mathrm{N}$ and residue burning effects on infiltration, biological properties and physical properties of intensively tilled silt-loam soil. Soil Tillage Research. 84, 154-167, 2005.

ZIEGLER, A.D.; BRUUN, T.B.; GUARDIOLA-CLARAMONTE, M.; GIAMBELLUCA, T.W.; LAWRENCE, D.; THANH LAM, N. Environmental consequences of the demise in swidden cultivation in Montane mainland Southeast Asia: hydrology and geomorphology. Human Ecology, v.37, p.361373, 2009. 